\title{
ON THE HOMOLOGY OF WEIGHTED HOMOGENEOUS MANIFOLDS
}

\author{
KAI WANG
}

\begin{abstract}
In this note we produce a formula for the rank of the homology groups of weighted homogeneous manifolds which is derived from Milnor and Orlik's formula. However, our formula is easier to compute if the weights have many repetitions.
\end{abstract}

Let $f(z)$ be a polynomial function in $m$ complex variables such that $f(0)=0$ and 0 is an isolated singular point. Let $K$ be the intersection of the hypersurface $f^{-1}(0)$ with a sufficiently small sphere $S^{2 m-1}$ centered at 0 . $K$ is a closed $(m-3)$-connected manifold of dimension $2 m-3$. The mapping $\Phi(\mathbf{z})=f(\mathbf{z}) /|f(\mathbf{z})|$ from $S^{2 m-1}-K$ to the unit circle is the projection map of a smooth fiber bundle. The fiber $F=\Phi^{-1}(1) \in S^{2 m-1}-K$ is a smooth, parallelizable $2(m-1)$-manifold. The Wang sequence corresponding to the above fibration reduces for $m \geqslant 3$ to the following short exact sequence:

$$
0 \rightarrow H_{m-1} K \rightarrow H_{m-1} F^{I^{*}-h^{*}}{ }^{\rightarrow} H_{m-1} F \rightarrow H_{m-2} K \rightarrow 0 .
$$

Here $I$ is the identity map of the fiber $F$ and $h: F \rightarrow F$ is the characterisitc map of the fibration. Thus the matrix $H$ of the map $h_{*}: H_{m-1} F \rightarrow H_{m-1} F$ contains all information about the homology of the $(m-3)$-connected manifold $K$. Denote its characteristic polynomial by $\Delta(t)=\operatorname{det}(t I-H)$. Brieskorn [1] computed $\Delta(t)$ for manifolds defined by polynomials of the form

$$
\cdot f(\mathbf{z})=z_{1}^{a_{1}}+z_{2}^{a_{2}}+\cdots+z_{m}^{a_{m}} .
$$

The references to above results are [1], [2].

Next, we will describe Milnor and Orlik's result on $\Delta(t)$ in [3] for weighted homogeneous polynomials.

DEFINITION 1. Let $w_{1}, \ldots, w_{m}$ be positive rational numbers. The polynomial $f\left(z_{1}, z_{2}, \ldots, z_{m}\right)$ is weighted homogeneous of type $\left(w_{1}, \ldots, w_{m}\right)$ if it can be expressed as a linear combination of monomials $z_{1}^{i_{1}} z_{2}^{i_{2}} \cdots z_{m}^{i_{m}}$ for which

$$
i_{1} / w_{1}+i_{2} / w_{2}+\cdots+i_{m} / w_{m}=1
$$

To each monic polynomial $\left(t-\alpha_{1}\right) \cdots\left(t-\alpha_{k}\right)$ with $\alpha_{1}, \ldots, \alpha_{k} \in C^{*}$ assign the divisor

Received by the editors January 18, 1977 and, in revised form, June 13, 1977.

AMS (MOS) subject classifications (1970). Primary 57D45, 57D60.

Key words and phrases. Hypersurface, characteristic polynomial, divisors.

() American Mathematical Society 1978 


$$
\operatorname{divisor}\left[\left(t-\alpha_{1}\right) \cdots\left(t-\alpha_{k}\right)\right]=\left\langle\alpha_{1}\right\rangle+\cdots+\left\langle\alpha_{k}\right\rangle
$$

thought of as an element of the integral group ring $Z C^{*}$. In particular, let

$$
\Lambda_{n}=\operatorname{divisor}\left(t^{n}-1\right)=\langle 1\rangle+\langle\xi\rangle+\cdots+\left\langle\xi^{n-1}\right\rangle
$$

where $\xi=\exp (2 \pi i / n)$. For integers $a_{1}, a_{2}, \ldots, a_{k}$ denote by $\left[a_{1}, a_{2}, \ldots, a_{k}\right]$ their least common multiple. For convenience, we let $[\varnothing]=1$ where $\varnothing$ is the empty set.

THEOREM 2 [3]. Let $f\left(z_{1}, z_{2}, \ldots, z_{m}\right)$ be a weighted homogeneous polynomial of type $\left(w_{1}, w_{2}, \ldots, w_{m}\right)$ having an isolated critical point at the origin. Express the weights in irreducible form as $w_{i}=u_{i} / v_{i}, i=1,2, \ldots, m$. Then the characteristic polynomial $\Delta(t)$ of the linear transformation

$$
h_{*}: H_{m-1} F \rightarrow H_{m-1} F
$$

is determined by

$$
\text { divisor } \Delta(t)=\sum_{I}(-1)^{m-s} \frac{w_{i_{1}} \cdots w_{i_{s}}}{\left[u_{i_{1}}, \ldots, u_{i_{s}}\right]} \Lambda_{\left[u_{i_{1}}, \ldots, u_{i_{s}}\right]}
$$

where I ranges over all $2^{m}$ subsets $\left\{i_{1}, \ldots, i_{s}\right\}$ of $\{1,2, \ldots, m\}$.

COROLlaRy 3 [3]. Let $\kappa$ be the rank of $H_{m-2} K$. Then

$$
\kappa=\sum_{I}(-1)^{m-s} \frac{w_{i_{1}} \cdots w_{i_{s}}}{\left[u_{i_{1}}, \ldots, u_{i_{s}}\right]}
$$

where I ranges over all $2^{m}$ subsets $\left\{i_{1}, \ldots, i_{s}\right\}$ of $\{1,2, \ldots, m\}$.

Thus $\Delta(t)$ and $\kappa$ may be computed explicitly in terms of the weights $\left(w_{1}, \ldots, w_{m}\right)$. It is easy to see that if $m$ is very large, then the computation of $\Delta(t)$ and $\kappa$ via formula (1) and (2) are very complicated. The purpose of this note is to offer another formula for $\kappa$ which is much easier to compute if there are many duplicates in the given weights $\left(w_{1}, \ldots, w_{m}\right)$.

Let $w_{1}, \ldots, w_{m}$ be positive rational numbers and let $w_{i}=u_{i} / v_{i}, i=$ $1, \ldots, m$ be the irreducible representations of $w_{i}$ as fractions of positive integers. We write $\left\{u_{1}, \ldots, u_{m}\right\}$ as $\left\{a_{1}, \ldots, a_{n} ; m(1), \ldots, m(n)\right\}$ where $\left\{a_{1}, \ldots, a_{n}\right\}$ is the complete subset of distinct elements of $\left\{u_{1}, \ldots, u_{m}\right\}$ and $m(i)$ is the multiplicity of $a_{i}$. Let $\mathbb{Q}=\left\{a_{1}, \ldots, a_{m}\right\}$. A subset $\left\{a_{i}, \ldots, a_{i_{s}}\right\}$ of $\mathbb{Q}$ is said to be saturated if there is no $a_{j} \notin\left\{a_{i_{1}}, \ldots, a_{i_{s}}\right\}$ so that $\left[a_{j}, a_{i}, \ldots\right.$, $\left.a_{i_{s}}\right]=\left[a_{i_{1}}, \ldots, a_{i_{s}}\right]$. The empty set $\varnothing$ is saturated since $[\varnothing]=1$.

Let $\delta$ be the collection of all saturated subsets of $Q$. Let $d=\left[a_{1}, \ldots, a_{n}\right]$. For $I \in \mathcal{S}$, let

$$
\mu(I)=\sum(-1)^{|J|} \frac{d}{[I, J]}
$$

where $J$ ranges over all subsets of $Q-I$ including $\varnothing$, and let $\mathscr{W}(I)=\left\{w_{i} \mid u_{i}\right.$ $\in I\}$ and $\tau(I)=\sum m(i)$ where $i$ ranges over all $i$ such that $a_{i} \in I$. Note that W $(I)$ includes all the repetitions.

With the notations as above, we have 


\section{THEOREM 4.}

$$
\kappa=\frac{1}{d}\left\{\sum_{I \in \mathcal{S}}(-1)^{m-\tau(I)} \mu(I) \prod_{w_{i} \in \mho(I)}\left(w_{i}-1\right)\right\} .
$$

Here we use the convention that if $I=\varnothing, \Pi_{w_{i} \in w(I)}\left(w_{i}-1\right)=1$. First, we need the following:

LEMMA 5. Let $u_{1}, u_{2}, \ldots, u_{m}$ be $m$ given positive integers and let $x_{1}, x_{2}, \ldots, x_{m}$ be $m$ independent variables. Then

(4) $\frac{1}{d}\left\{\sum_{k=1}^{d}(-1)^{m-|\Gamma(k)|} \prod_{i \in \Gamma(k)}\left(x_{i}-1\right)\right\} \equiv \sum_{I}(-1)^{m-s} \frac{x_{i_{1}} \cdots x_{i_{s}}}{\left[u_{i_{1}}, \ldots, u_{i_{s}}\right]}$

where $I$ ranges over all $2^{m}$ subsets of $\{1,2, \ldots, m\}, d=\left[u_{1}, \ldots, u_{m}\right]$ and

$$
\Gamma(k)=\left\{i \mid k \equiv 0 \quad\left(\bmod u_{i}\right)\right\},
$$

and "三" means "equal for all values of the variables."

Proof. Let $f\left(x_{1}, \ldots, x_{m}\right)=d \Sigma_{I}(-1)^{s} x_{i_{1}} \cdots x_{i_{s}} /\left[u_{i_{1}}, \ldots, u_{i_{s}}\right]$,

$$
g_{k}\left(x_{1}, \ldots, x_{m}\right)=\prod_{i \in \Gamma(k)}\left(x_{i}-1\right)
$$

and

$$
g\left(x_{1}, \ldots, x_{m}\right)=\sum_{k=1}^{d}(-1)^{|\Gamma(k)|} g_{k}\left(x_{1}, \ldots, x_{m}\right) .
$$

It is obvious that (1) holds if and only if $f\left(x_{1}, \ldots, x_{m}\right) \equiv g\left(x_{1}, \ldots, x_{m}\right)$. Since $g\left(x_{1}, \ldots, x_{m}\right)$ is also a linear combination of $x_{i_{1}} \cdots x_{i_{s}}$ which has $2^{m}$ terms, it is sufficient to show $f\left(x_{1}, \ldots, x_{m}\right)=g\left(x_{1}, \ldots, x_{m}\right)$ for $2^{m}$ different values of $\left\{x_{1}, \ldots, x_{m}\right\}$. For any subset $I$ of $\{1,2, \ldots, m\}$, let

$$
x_{i}= \begin{cases}0 & \text { if } i \notin I \\ 1 & \text { if } i \in I .\end{cases}
$$

Then

$$
\begin{aligned}
f\left(x_{1}, \ldots, x_{m}\right) & =\sum_{\left\{i_{1}, \ldots, i_{s}\right\} \subset I}(-1)^{s} \frac{d}{\left[u_{i_{1}}, \ldots, u_{i_{s}}\right]} \\
& =\operatorname{card}\left\{1 \leqslant k \leqslant d \mid k \neq 0\left(\bmod u_{i}\right) \text { for all } i \in I\right\} .
\end{aligned}
$$

On the other hand,

$$
g_{k}\left(x_{1}, \ldots, x_{m}\right)= \begin{cases}0 & \text { if } \Gamma(k) \cap I \neq \varnothing \\ (-1)^{\Gamma(k)} & \text { if } \Gamma(k) \cap I=\varnothing\end{cases}
$$

Hence

$$
\begin{aligned}
g\left(x_{1}, \ldots, x_{m}\right) & =\sum_{\Gamma(k) \cap I=\varnothing}(-1)^{|\Gamma(k)|+|\Gamma(k)|} \\
& =\operatorname{card}\{1 \leqslant k \leqslant d \mid \Gamma(k) \cap I=\varnothing\} .
\end{aligned}
$$


However, it is easy to see that $\Gamma(k) \cap I=\varnothing$ if and only if $k \neq 0\left(\bmod u_{i}\right)$ for all $i \in I$. Now the lemma is clear.

Next, as before, let $\mathcal{Q}=\left\{a_{1}, \ldots, a_{n}\right\}$ be the complete set of distinct elements of $\left\{u_{1}, \ldots, u_{m}\right\}$ and let $\delta$ be the set of all saturated subsets and for $I \in \mathcal{S}$, let $\mathcal{Q}(I)=\left\{x_{i} \mid u_{i} \in I\right\}$. It is easy to see that every $\Gamma(k)$ is of the form $\mathcal{W}(I)$ for some $I \in \mathcal{S}$ and $\Gamma(k)=\mathscr{W}(I)$ if and only if $k$ is a multiple of [I] and $k$ is not a multiple of any other $[J], J \in \mathcal{S}$. It follows that the number of $k, 1 \leqslant k \leqslant d$, such that $\Gamma(k)=\mathscr{W}(I)$ is equal to $\mu(I)$. Thus we can rewrite equation (4) as

$$
\sum_{I}(-1)^{m-s} \frac{x_{i_{1}} \cdots x_{i_{s}}}{\left[u_{i_{1}}, \ldots, u_{i_{s}}\right]} \equiv \frac{1}{d} \sum_{I \in \mathcal{S}}(-1)^{m-|\mho(I)|} \mu(I) \prod_{x_{i} \in \mathscr{W}(I)}\left(x_{i}-1\right) .
$$

Hence we get (3) by substituting $x_{i}$ with $w_{i}$. This completes the proof of Theorem 4.

REMARK 6. The following is a short cut to compute $\mu(I)$. First, we order $\mathcal{\delta}$, the set of all saturated subsets, by requiring $\left[I_{s}\right]>\left[I_{s+1}\right], s=1,2, \ldots$ It is obvious that $I_{1}=\left\{a_{1}, \ldots, a_{n}\right\}$, and $\mu\left(I_{1}\right)=1$. Suppose that $\mu\left(I_{1}\right)$, $\mu\left(I_{2}\right), \ldots, \mu\left(I_{l}\right)$ have been computed. Then

$$
\mu\left(I_{l+1}\right)=d /\left[I_{l+1}\right]-\sum_{I_{j} \supset I_{l+1}} \mu\left(I_{j}\right)
$$

For any set of positive rational numbers $\mathscr{W}=\left\{w_{1}, \ldots, w_{m}\right\}$, let

$$
\kappa(\mathcal{W})=\sum(-1)^{m-s} \frac{w_{i_{1}} \cdots w_{i_{s}}}{\left[u_{i_{1}}, \ldots, u_{i_{s}}\right]}
$$

where $\left\{w_{i_{1}}, \ldots, w_{i_{s}}\right\}$ ranges over all $2^{m}$ subsets of $\left\{w_{1}, \ldots, w_{m}\right\}$ and $u_{i}$ are as in Theorem 2. Then the characteristic polynomial $\Delta(t)$ in Theorem 2 can be computed explicitly in terms of $\kappa(\mathscr{W}(I)), I \in \mathcal{S}$.

THEOREM 7. Let divisor $\Delta(t)=\Sigma_{I \in \delta} \varphi([I]) \Lambda_{[I]}$. Then

$$
\kappa(\mathcal{Q}(I))=(-1)^{m-\tau(I)}\left(\sum_{\substack{J \in \mathcal{S} \\ J \subset I}} \varphi([J])\right)
$$

Proof. By Theorem 2

$$
\varphi([J])=\sum(-1)^{m-s} \frac{w_{i_{1}} \cdots w_{i_{s}}}{\left[u_{i_{1}}, \ldots, u_{i_{s}}\right]}
$$

where $\left\{w_{i_{1}}, \ldots, w_{i_{s}}\right\}$ ranges over all subsets of $\left\{w_{1}, \ldots, w_{m}\right\}$ such that $\left[u_{i}, \ldots, u_{i s}\right]=[J]$. Hence 


$$
\begin{aligned}
(-1)^{m-\tau(I)}\left(\sum_{\substack{J \in S \\
J \subset I}} \varphi([J])\right)=\sum_{\substack{J \in S \\
J \subset I}}\left(\sum_{\left[u_{i}, \ldots, u_{i}\right]=[J]}(-1)^{\tau(I)-s} \frac{w_{i_{1}} \cdots w_{i_{s}}}{\left[u_{i_{1}}, \ldots, u_{i_{s}}\right]}\right) \\
=\sum(-1)^{\tau(I)-s} \frac{w_{i_{1}} \cdots w_{i_{s}}}{\left[u_{i_{1}}, \ldots, u_{\left.i_{s}\right]}\right.} \\
\quad\left(\text { where }\left\{w_{i_{1}}, \ldots, w_{i_{s}}\right\} \text { ranges over all } 2^{\tau(I)} \text { subsets of } \mathcal{W}(I)\right) \\
=\kappa(W(I)) .
\end{aligned}
$$

Numerical examples. 1. $f\left(z_{1}, \ldots, z_{m}\right)=z_{1}^{d}+\cdots+z_{m}^{d}$.

It is easy to see that $\delta=\{\varnothing,\{d\}\}$ and $\mu(\{d\})=1, \mu\{\varnothing\}=d-1$. Hence

$$
\kappa=\frac{1}{d}\left((d-1)^{n}+(-1)^{n}(d-1)\right) \text {. }
$$

2. $f\left(z_{1}, \ldots, z_{8}\right)=z_{1}^{3}+z_{2}^{3}+z_{3}^{5}+z_{4}^{5}+z_{5}^{15}+z_{2} z_{6}+z_{4} z_{7}+z_{5} z_{8}$.

It is easy to see that $f$ has an isolated singularity at 0 and is weighted homogeneous of type $(3,3,5,5,15,3 / 2,5 / 4,15 / 14)$. It is easy to see that $\mathcal{S}=\{\varnothing,\{3\},\{5\},\{3,5,15\}\}, d=15, \mu(\{3,4,15\})=1, \mu(\{5\})=2, \mu(\{3\})$ $=4, \mu(\varnothing)=8$. Then

$$
\begin{aligned}
\kappa=\frac{1}{15}[8 & -4(3-1)^{2}\left(\frac{3}{2}-1\right)-2(5-1)^{2}\left(\frac{5}{4}-1\right) \\
& \left.+(3-1)^{2}\left(\frac{3}{2}-1\right)(5-1)^{5}\left(\frac{5}{4}-1\right)(15-1)\left(\frac{15}{14}-1\right)\right]=0 .
\end{aligned}
$$

Note that $\mathscr{W}(\{5\})=\{5,5,5 / 4\}, \quad \mathscr{W}(\{3\})=\{3,3,3 / 2\}$. It is easy to compute that

$$
\kappa(\mathscr{W}(\{5\}))=\kappa(\mho(\{3\}))=0 .
$$

It follows that $\varphi(1)=\varphi(15)=1, \varphi(3)=\varphi(5)=-1$. Hence divisor $\Delta(t)=\Lambda_{1}$ $-\Lambda_{3}-\Lambda_{5}+\Lambda_{15}$ or equivalently

$$
\Delta(t)=\frac{(t-1)\left(t^{15}-1\right)}{\left(t^{3}-1\right)\left(t^{5}-1\right)} .
$$

Consequently $\Delta(1)=1$. This implies the weighted homogeneous manifold $K$ defined by $f$ as described at the beginning of this note is a homotopy 15-sphere.

\section{REFERENCES}

1. E. Brieskorn, Beispiele zur Differential topologie von Singularitaten, Invent. Math. 2 (1966), 1-14.

2. J. Milnor, Singular points of complex hypersurfaces, Ann. of Math. Studies, No. 61, Princeton Univ. Press, Princeton, N. J., 1968.

3. J. Milnor and P. Orlik, Isolated singularities defined by weighted homogeneous polynomials, Topology 9 (1970), 385-393.

Department of Mathematics, State University of New York at Buffalo, Buffalo, New YORK 14214 